Research Article

\title{
Effects of Macrofungal Polysaccharides Combined with Vemurafenib on Melanoma and Its Associated Mechanism
}

\author{
Wenya Wu, ${ }^{1}$ Zhigang Su, ${ }^{2}$ and Shasha Fan $\mathbb{D}^{3}$ \\ ${ }^{1}$ Department of Dermatology, The Second Affiliated Hospital of Soochow University, Suzhou, China \\ ${ }^{2}$ Department of Urology, The Second Affiliated Hospital of Soochow University, Suzhou, China \\ ${ }^{3}$ Department of Dermatology, Zhejiang Provincial People's Hospital, People's Hospital of Hangzhou Medical College, \\ Hang Zhou, China
}

Correspondence should be addressed to Shasha Fan; fanshasha@hmc.edu.cn

Received 13 May 2019; Accepted 11 July 2019; Published 24 October 2019

Guest Editor: Jingxiao Chen

Copyright (c) 2019 Wenya Wu et al. This is an open access article distributed under the Creative Commons Attribution License, which permits unrestricted use, distribution, and reproduction in any medium, provided the original work is properly cited.

Fungal polysaccharides have demonstrated various biological functions such as antitumor, immune regulation, and antioxidant activities. It has also been reported to be beneficial in reshaping the immune system's surveillance on tumor cells and in helping the immune system kill tumor cells. In this study, a melanoma mouse model was constructed, and a macrofungal polysaccharide (MFPS) extracted from Pleurotus ostreatus combined with Vemurafenib monoclonal antibody was used to study their effects against melanoma and its antitumor mechanism by using the lactate dehydrogenase release assay, enzyme-linked immunosorbent assay, and flow typing assay. Results indicated that MFPS enhanced the inhibitory effect of Vemurafenib on tumor growth in melanoma-bearing mice and the secretion of cytokines IFN- $\gamma$ and IL-12 in PBMCs of melanoma-bearing mice. In addition, the combination of MFPS1 and Vemurafenib can enhance the immunomodulatory activity of melanoma-bearing mice as well as elicit the activation and proliferation of $\mathrm{B}$ cells and $\mathrm{T}$ cells.

\section{Introduction}

Traditional chemotherapy targets tumors, but it is often accompanied with obvious side effects including causing toxic effects in healthy organs. In recent years, with the continuous advancement in research related to cancer treatment, the combination of immunotherapy and chemotherapy has received more and more attention. Some clinical trials with combined immunotherapy and chemotherapy have shown that patients who received chemotherapy with subsequent reception of immunotherapy had better clinical outcomes than patients who only received chemotherapy $[1,2]$. Other preclinical and clinical studies have also shown that chemotherapy can improve the efficacy of immunotherapy through various mechanisms [3]. Chemotherapy can not only overcome partial immunosuppression but also heighten the cross-expression of tumor antigens and enhance the infiltration of immune cells into the tumor tissue.
Fungal polysaccharides are a class of polysaccharides that have received an increasing amount of attention in recent years. Fungal polysaccharides have been shown to have a variety of biological activities such as antitumor, immune regulation, and antioxidant $[4,5]$. Its biological activity depends on the structure, molecular weight, monosaccharide unit, and type of links in the polysaccharide. Fungal polysaccharides consist of different types of polysaccharides found in fungal cells, from high molecular weight to low molecular weight and from homopolysaccharides to heteropolysaccharides with different linkage types [6, 7].

In the advanced stages of cancer, tumor cells usually enter the blood circulation and begin to metastasize. At this stage, tumor cells can circulate into various organs and cells of the body through the blood. The blood circulation system contains certain components of the immune system, and these components typically can play an immune surveillance role in the early stages of cancer; however, with an advanced 


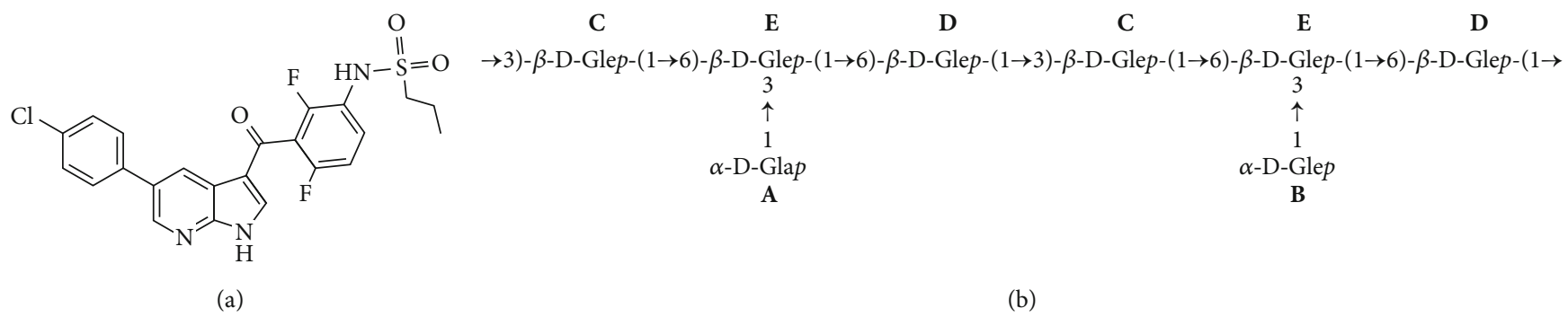

FIGURE 1: The molecular formula of Vemurafenib and the water-soluble component of macrofungal heteropolysaccharide (MFPS1): (a) Vemurafenib; (b) MFPS1.

stage of cancer, the immune system has been proved to have lost the role of immune surveillance and unable to recognize cancer cells [8]. Therefore, remodeling the immune system's surveillance of tumor cells plays a key role in the treatment of cancer. Studies have demonstrated that fungal polysaccharides can be beneficial in the reshaping of the immune system to monitor tumor cells and in helping the immune system kill tumor cells [9-11].

Vemurafenib is a mutant-selective serine/threonine kinase inhibitor specifically designed to inhibit V-RAF mutations of the murine sarcoma viral oncogene homolog B1 (BRAF) gene in the mitogen-activated protein kinase/extracellular signal-regulated kinase signaling pathway (MAPK/ERK) that is often associated with cancer development. BRAF mutations are typically found in melanoma, colorectal cancer, papilloma, thyroid cancer, non-smallcell lung cancer, and ovarian cancer [12]. Studies have shown that almost $50 \%$ of patients with malignant melanoma contain BRAF mutations [13]. With that, Vemurafenib was approved in 2011 for the treatment of advanced malignant melanoma with BRAF mutations [14]. The structure for Vemurafenib is shown in Figure 1(a).

The clinical trial of Vemurafenib began in 2006. In a phase I trial, 32 patients reported an objective response rate of $81 \%$ after being treated with Vemurafenib. The next phase III trial showed that patients treated with Vemurafenib had a significant improvement in the survival rate compared with patients receiving dacarbazine, and the risk of death was reduced by $63 \%$ [15].

In this study, we focus on the combined effects of macrofungal polysaccharides (MFPS) extracted from Pleurotus ostreatus and Vemurafenib monoclonal antibody on melanoma and its antitumor mechanism. We also attempted to uncover the antimelanoma-associated immune pathway affected by the combination use of MFPS and Vemurafenib.

\section{Materials and Methods}

2.1. Experimental Materials. Human melanoma MeWo cell lines were purchased from the Shanghai Cell Bank of the Chinese Academy of Sciences. Fetal bovine serum (FBS), RPMI-1640 medium, and EDTA were purchased from Gibco. The lymphocyte separation solution and lactate dehydrogenase release assay (LDH) kit were purchased from Japan Tongren Co. Ltd. The enzyme-linked immunosorbent assay kit for measurement of IL-12 and INF- $\gamma$ was purchased from Xinbosheng Biotechnology Co. Ltd. $\mathrm{SPF}$ male BALB/c/nu nude mice with body weight $18 \pm 2$ $\mathrm{g}$ were purchased from Shanghai Slack Laboratory Animal Center. CD45-percp, CD4-fitc, CD19-apc, CD34-fitc, and CD335-apc antibodies were all purchased from BD Biotechnology Co. Ltd. Other biochemical reagents were purchased from Sinopharm Group.

2.2. Isolation and Purification of Macrofungal Polysaccharide. The mushroom fruiting bodies were washed with sterilized double distilled water, pulverized, and then soaked at $90^{\circ} \mathrm{C}$, with $4 \% \mathrm{NaOH}$ for $6 \mathrm{~h}$. The extract was filtered with Whatman No. 1 filter paper, and the filtrate was mixed with $80 \%$ ethanol at a volume ratio of $1: 5$ and then allowed to stand at $4^{\circ} \mathrm{C}$ overnight to precipitate polysaccharide. The precipitated crude polysaccharide was centrifuged at $4^{\circ} \mathrm{C}, 8000 \mathrm{rpm}$ for 40 minutes, and then washed with $80 \%$ and $90 \%$ ethanol and acetone, respectively, followed by the collection of high alkaline crude polysaccharide solution having a $\mathrm{pH}$ of 11 . The solution was then dialyzed in the double distilled water against a membrane with a molecular weight of $10000 \mathrm{~g} / \mathrm{mol}$ at $4^{\circ} \mathrm{C}$ for 3 days to remove compounds of low molecular weight and excess $\mathrm{NaOH}$. During the dialysis, the alkali-soluble fraction precipitates and the water-soluble components remain in the solution. The macrofungal heteropolysaccharide (MFPS1) was obtained after centrifugation and then lyophilized and stored [16]. The molecular formula of MFPS1 obtained in this way is shown in Figure 1(b).

\subsection{Construction of Human Melanoma MeWo Cell-Bearing} Mice. BALB/c nude mice of 6 to 12 weeks old were used to construct human melanoma tumor-bearing mice. $3 \times 10^{6}$ human melanoma MeWo cells in a logarithmic growth phase were injected subcutaneously into the right ankle of nude mice. When the tumor grows to at least $100-300 \mathrm{~mm}^{3}$, the mice were used for in vivo experiments [17].

2.4. Drug Treatment for Human Melanoma Tumor-Bearing Mice. When the tumor of human melanoma-bearing mice grows to at least $100-300 \mathrm{~mm}^{3}$, the tumor-bearing mice were divided into the following 4 groups (6 in each group) for drug treatment: (1) control group: normal diet for 12 days; 
(2) Vemurafenib group: Vemurafenib and MFPS1 were dissolved in DMSO and saline solution, respectively, and then orally administered at $50 \mathrm{mg} / \mathrm{kg} /$ day for 12 days [18]; (3) low-dose MFPS1+Vemurafenib group: Vemurafenib was orally administered at $50 \mathrm{mg} / \mathrm{kg} / \mathrm{day}$, and the MFPS1 was also administered at $100 \mathrm{mg} / \mathrm{kg} /$ day at the same time for 12 days; (4) high-dose MFPS1+Vemurafenib group: Vemurafenib was orally administered at $50 \mathrm{mg} / \mathrm{kg} / \mathrm{day}$, and the MFPS1 was also administered at $150 \mathrm{mg} / \mathrm{kg} /$ day at the same time for 12 days. The control group was given the same amount of saline or DMSO solution per day as in the experimental group. The tumor size was measured every 3 days and weighed; the tumor growth curve was drawn afterward.

2.5. Isolation of Mouse Peripheral Blood Mononuclear Cells (PBMCs). Human melanoma-bearing mice were anesthetized with an appropriate amount of pentobarbital sodium anesthetic. Blood was then taken from the eyelids, $1 \mathrm{ml} /$ mouse, and was collected into an EDTA anticoagulant tube, and then, the PBMCs were isolated by the Ficoll method. The isolated PBMCs were used for the lactate dehydrogenase (LDH) release assay and flow cytometry.

2.6. LDH Experiment. The PBMCs of 4 groups of melanoma-bearing mice were separately prestimulated by adding corresponding drugs; that is, DMSO was added to PBMCs from normal mice as a control, Vemurafenib $(2 \mu \mathrm{g} / \mathrm{ml})$ was added to PBMCs from Vemurafenib mice, Vemurafenib $(2 \mu \mathrm{g} / \mathrm{ml})$ and MFPS1 $(4 \mu \mathrm{g} / \mathrm{ml})$ were added to PBMCs from low-dose MFPS1 combined with Vemurafenib mice, and Vemurafenib $(2 \mu \mathrm{g} / \mathrm{ml})$ and MFPS1 $(8 \mu \mathrm{g} / \mathrm{ml})$ were added to PBMCs from high-dose MFPS1 combined with Vemurafenib mice. After 24 hours of incubation, PBMCs and human melanoma MeWo cells were subjected to LDH experiments at $1: 1,5: 1$, and $10: 1$ effective target ratios, respectively.

2.7. Enzyme-Linked Immunosorbent Assay (ELISA). The PBMCs of 4 groups of melanoma-bearing mice were prestimulated according to the method of LDH experiment, the supernatant was collected after 24 hours of incubation, and ELISA was performed to detect cytokines IFN- $\gamma$ and IL-12 and IL- 6 and TNF- $\alpha$.

2.8. Immunophenotyping. The PBMCs of 4 groups of melanoma-bearing mice were prestimulated according to the method of LDH experiment, and after 24 hours of incubation, the cells were collected in precooled PBS and cultured with monoclonal antibodies: CD45-percp, CD4-fitc, CD19apc, CD34-fitc, and CD335-apc. After incubation at $4^{\circ} \mathrm{C}$ for $3 \mathrm{~h}$, the expression-dependent cell population was analyzed by flow cytometry.

2.9. Data Analysis. Data were expressed as the mean \pm standard deviation. Software Origin8.5 was used for oneway variance analysis, and statistical significance was observed when $p<0.05$.

\section{Results}

3.1. MFPS1 Enhanced the Inhibitory Effect of Vemurafenib on Tumor Growth in Melanoma-Bearing Mice. The combined effects of MFPS1 and Vemurafenib on the tumor growth of melanoma-bearing mice were studied in the human melanoma MeWo cell-bearing mice. The results showed that both low-dose and high-dose MFPS1 enhanced the inhibitory effect of Vemurafenib on tumor growth in melanomabearing mice (Figure 2(a)). On the 12th day after drug administration, the tumor volumes of the tumor-bearing mice in the normal group, the Vemurafenib group, the lowdose MFPS1+Vemurafenib group, and the high-dose MFPS1+Vemurafenib group were $515.23 \pm 19.14 \mathrm{~mm}^{3}$, $411.9 \pm 20.14 \mathrm{~mm}^{3}, 387.02 \pm 17.14 \mathrm{~mm}^{3}$, and $362.02 \pm 19.83$ $\mathrm{mm}^{3}$, respectively. Compared with the normal group, the tumor volumes of the tumor-bearing mice in the Vemurafenib group, in the low-dose MFPS1+Vemurafenib group, and the high-dose MFPS1+Vemurafenib group were significantly reduced $(p<0.05)$. Moreover, compared with the Vemurafenib group, the tumor volumes in the low-dose MFPS1+Vemurafenib group and in the high-dose MFPS1 + Vemurafenib group were both significantly smaller $(p<0.05)$, and the high-dose MFPS1 group was superior to the low-dose MFPS1 group in this regard as shown in Figure 2(b).

3.2. In Vivo Experiments, MFPS1 Enhanced the Killing Effect of Vemurafenib on Human Melanoma MeWo Cells. The PBMCs of each group of melanoma-bearing mice were isolated and then prestimulated by the corresponding drugs; after that, the lactate dehydrogenase release assay (LDH) was then conducted for the in vivo experiment. The results from in vivo experiments indicated that MFPS1 enhanced the killing effect of Vemurafenib on human melanoma MeWo cells (Figure 3(a)).

When the ratio of immune cells and tumor cells (target ratio) was $5: 1$, in the normal group, the Vemurafenib group, the low-dose MFPS1+Vemurafenib group, and the high-dose MFPS1+Vemurafenib, the killing rates of MeWo cells were $21.56 \pm 0.21,62.98 \pm 2.21,67.1 \pm 3.81$, and $69.66 \pm 3.31$, respectively. Compared with the normal group, the killing effects on the human melanoma MeWo cells in the Vemurafenib group, in the low-dose MFPS1+Vemurafenib group, and in the high-dose MFPS1+Vemurafenib group were significantly higher $(p<0.01)$. Compared with the Vemurafenib group, the killing effects on the human melanoma MeWo cells in the low-dose MFPS1+Vemurafenib group and in the high-dose MFPS1+Vemurafenib group were significantly heightened $(p<0.05)$, and the high-dose MFPS1 group was superior to the low-dose MFPS1 group as suggested in Figure 3(b).

When the ratio of immune cells and tumor cells (target ratio) was $10: 1$, in the normal group, the Vemurafenib group, the low-dose MFPS1+Vemurafenib group, and the high-dose MFPS1+Vemurafenib, the killing rates of MeWo cells were $20.35 \pm 0.98,85.75 \pm 3.98,93 \pm 3.72$, and $96.66 \pm$ 3.966 , respectively. And compared with the normal group, the killing effects on the human melanoma MeWo cells in 


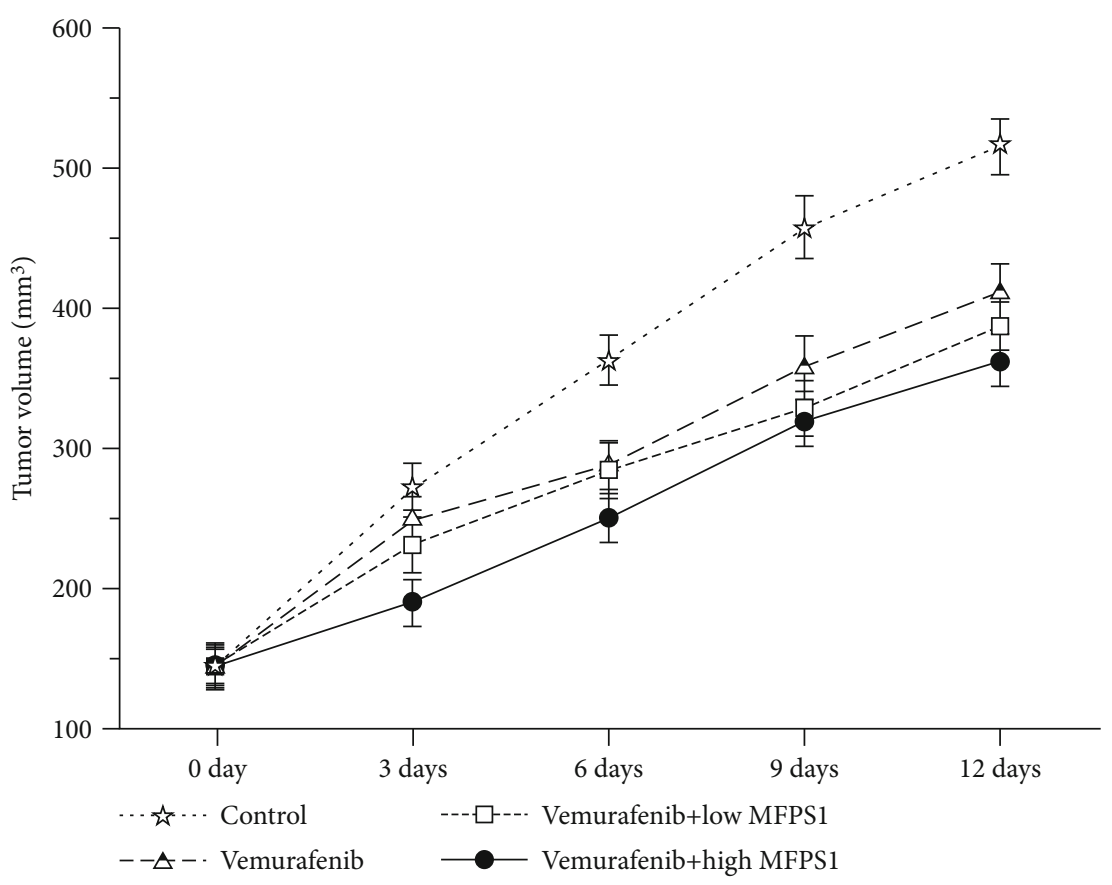

(a)

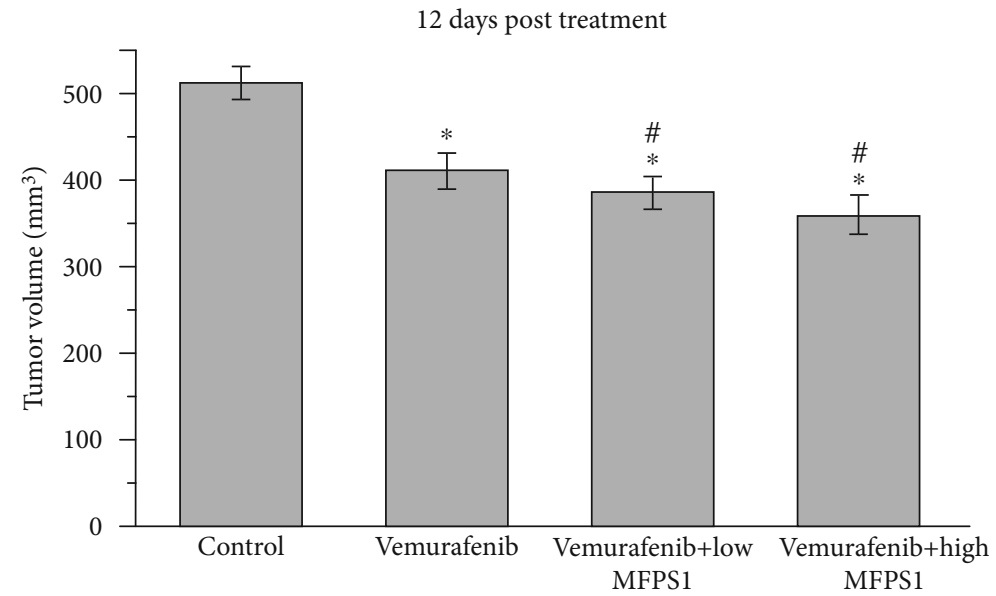

(b)

FigURE 2: MFPS1 enhanced the inhibitory effect of Vemurafenib on tumor growth in melanoma-bearing mice: (a) tumor growth curve of human melanoma MeWo cell-bearing mice 12 days after drug treatment; (b) tumor size of human melanoma MeWo cell-bearing mice at 12 days of drug treatment. * represents the normal group as the control: ${ }^{*} p<0.05$ and ${ }^{* *} p<0.01$; \# represents the Vemurafenib group as the control: ${ }^{\#} p<0.05$ and ${ }^{\# \#} p<0.01$.

the Vemurafenib group, in the low-dose MFPS1+Vemurafenib group, and in the high-dose MFPS1+Vemurafenib group were significantly higher $(p<0.01)$. When compared with the Vemurafenib group, the killing effects on the human melanoma MeWo cells in the low-dose MFPS1+Vemurafenib group and in the high-dose MFPS1+Vemurafenib group were significantly increased $(p<0.05)$, and the high-dose MFPS1 group was superior to the low-dose MFPS1 group (Figure 3(c)), which is similar to what was observed when the target ratio was $5: 1$.

3.3. In Vivo Experiments, MFPS1 Enhanced the CytokineSecreting Effect of Vemurafenib on PBMCs in MelanomaBearing Mice. To study the mechanism against melanoma by the combination of MFPS1 and Vemurafenib, the supernatant of PBMCs was collected after the tumor-bearing mice were prestimulated as described above, and the levels of cytokines interleukin-12 (IL-12) and interferon- $\gamma$ (IFN- $\gamma$ ) in the supernatant were measured. The results showed that the levels of IFN- $\gamma$ and IL-12 were both increased in the MFPS1-treated PBMCs (Figure 4).

The levels of IL-12 in PBMCs for the normal group, Vemurafenib group, low-dose MFPS+Vemurafenib group, and high-dose MFPS+Vemurafenib group were $391.5 \pm$ $2.97,449 \pm 1.92,490.35 \pm 24.5$, and $491 \pm 19$, respectively. Compared with the normal group, the levels of IL-12 in PBMCs in the Vemurafenib group, in the low-dose MFPS1 + Vemurafenib group, and in the high-dose MFPS1 


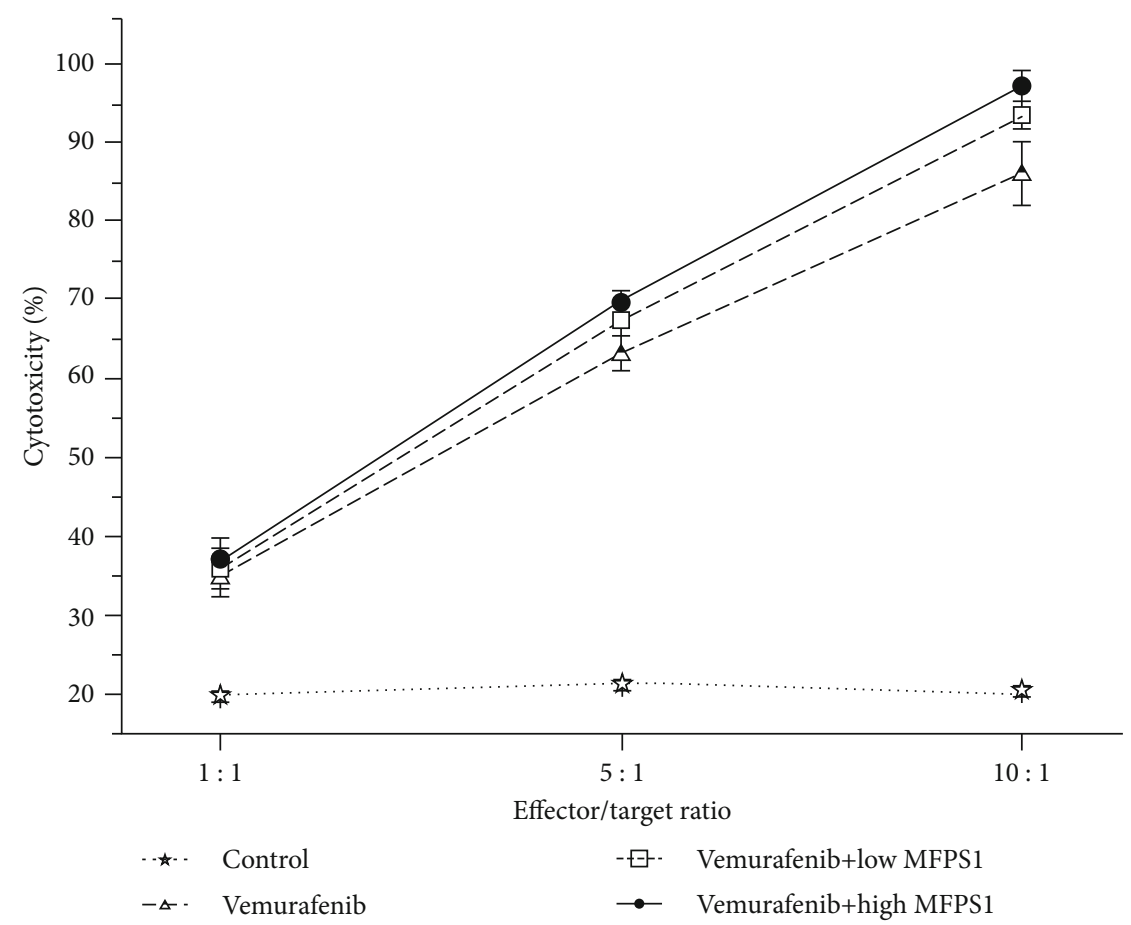

(a)

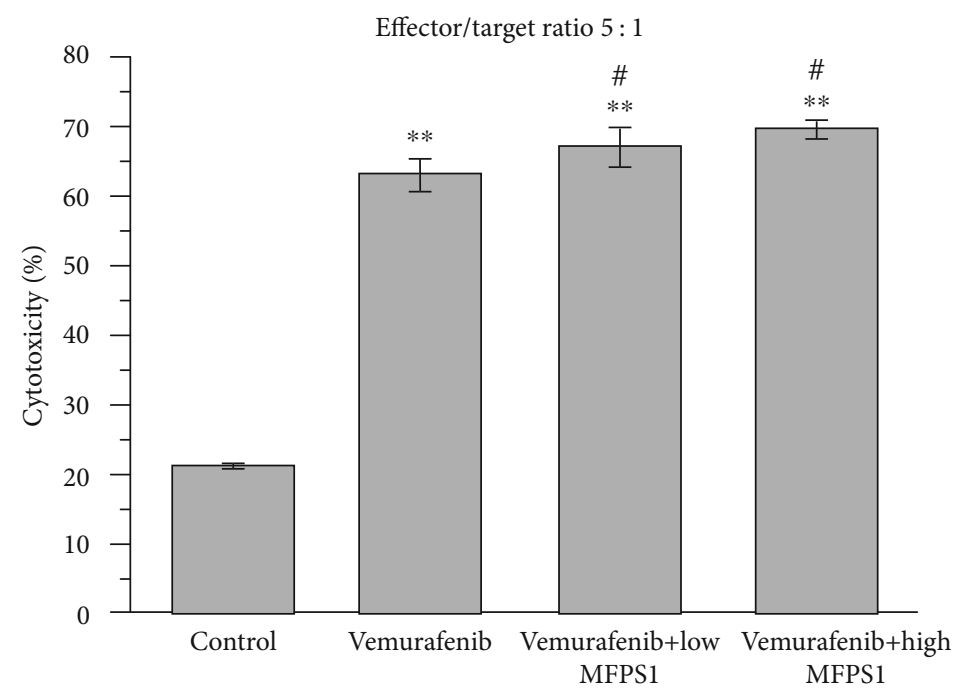

(b)

Figure 3: Continued. 


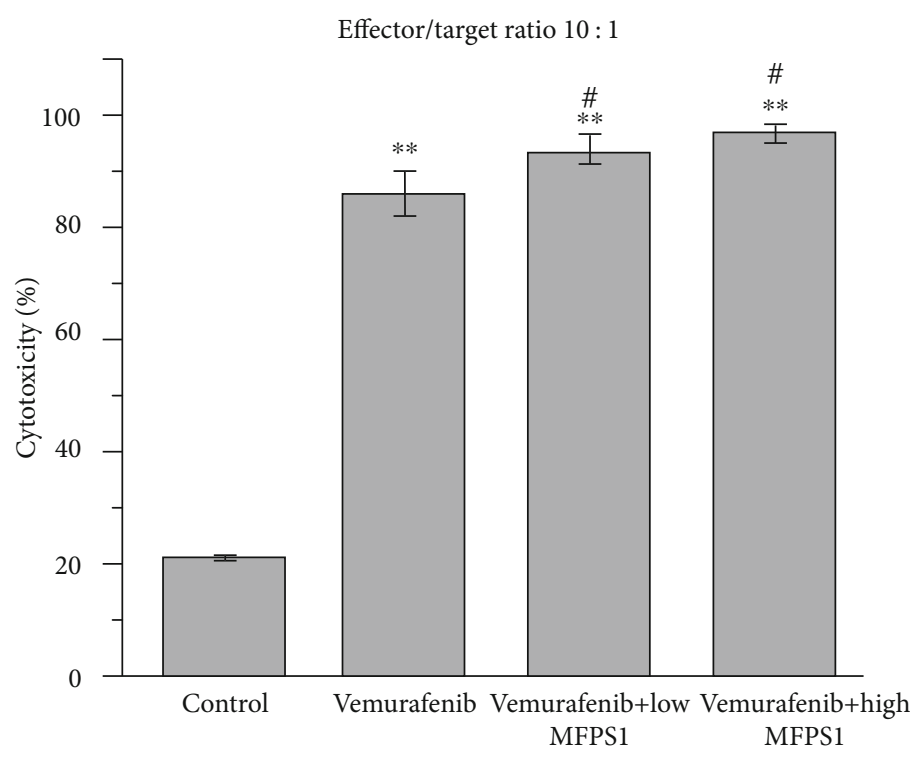

(c)

FIGURE 3: In vivo experiments, MFPS1 enhance the killing effect of Vemurafenib on human melanoma MeWo cells. (a) Killing effects on the human melanoma MeWo cells at target ratios of $1: 1,5: 1$, and $10: 1$ after drug treatment; (b) killing effects on the human melanoma MeWo cells at target ratio of 5:1 after drug treatment; (c) killing effects on the human melanoma MeWo cells at a target ratio of $10: 1$ after drug treatment. $*$ represents the normal group as the control: ${ }^{*} p<0.05$ and ${ }^{* *} p<0.01$; \# represents the Vemurafenib group as the control: $\# p<0.05$ and ${ }^{\# \#} p<0.01$.

+ Vemurafenib group were significantly increased $(p<0.01)$. Compared with the Vemurafenib group, the levels of IL-12 in PBMCs in the low-dose MFPS1+Vemurafenib group and in the high-dose MFPS1+Vemurafenib group were significantly higher $(p<0.05)$ (Figure $4(\mathrm{a})$ ).

The contents of INF- $\gamma$ in PBMCs for the normal group, Vemurafenib group, low-dose MFPS+Vemurafenib group, and high-dose MFPS+Vemurafenib group were $60.27 \pm$ $2.25,81.06 \pm 2.44,90.41 \pm 2.48$, and $91.333 \pm 1.86$, respectively. Compared with the normal group, the contents of INF- $\gamma$ in PBMCs in the Vemurafenib group, in the lowdose MFPS1+Vemurafenib group, and in the high-dose MFPS1+Vemurafenib group were significantly increased $(p<0.01)$. Compared with the Vemurafenib group, the contents of INF- $\gamma$ in PBMCs in the low-dose MFPS1+Vemurafenib group and in the high-dose MFPS1+Vemurafenib group were significantly higher $(p<0.05)$ (Figure $4(\mathrm{~b})$ ).

3.4. Effect of MFPS1 Combined with Vemurafenib on Immunomodulatory Activity in Melanoma-Bearing Mice. The activation of the immune system plays a very important role in tumor therapy. To further explore the antitumor mechanisms of MFPS1 in combination with Vemurafenib, we investigated the expression of immune cell-specific markers in PBMC cells. The results showed that the combination of MFPS1 and Vemurafenib enhanced the immunomodulatory effects of Vemurafenib on melanoma-bearing mice.

The lymphocyte contents in PBMCs for the normal group, Vemurafenib group, low-dose MFPS1+Vemurafenib group, and high-dose MFPS1+Vemurafenib group were
$29.2 \pm 1.34, \quad 37.87 \pm 2.85, \quad 45.28 \pm 1.33$, and $49.4 \pm 2.93$, respectively. Compared with the normal group, the lymphocyte contents in PBMCs in the Vemurafenib group, in the low-dose MFPS1+Vemurafenib group, and in the high-dose MFPS1+Vemurafenib group were significantly increased $(p<0.01)$. Compared with the Vemurafenib group, the lymphocyte contents in PBMCs in the low-dose MFPS1+Vemurafenib group and in the high-dose MFPS1+Vemurafenib group were significantly higher $(p<0.05)$ as indicated in Figure 5(a).

As shown in Figures 5(c) and 5(d), the content of mature $\mathrm{B}$ cells in PBMCs was increased after the combined treatment of MFPS1 and Vemurafenib, whereas the content of immature $\mathrm{B}$ cells was decreased. A similar trend was also observed for the $\mathrm{T}$ cell content (Figures 5(e) and 5(f)). Additionally, CD335+ cells were also found to increase after the combined treatment of MFPS1 and Vemurafenib (Figure 5(b)). Since CD335 is a cytotoxic activating receptor expressed in activated NK cells, the results above thus suggested that NK cells were also activated in PBMCs of MFPS1 and Vemurafenibtreated mice.

\section{Discussion}

Results in this study demonstrated that MFPS1 enhanced the inhibitory effect of Vemurafenib on tumor growth in melanoma-bearing mice and the secretion of cytokines IFN- $\gamma$ and IL-12 in PBMCs of melanoma-bearing mice. Moreover, the combination of MFPS1 and Vemurafenib can enhance the immunomodulatory activity of melanomabearing mice. 


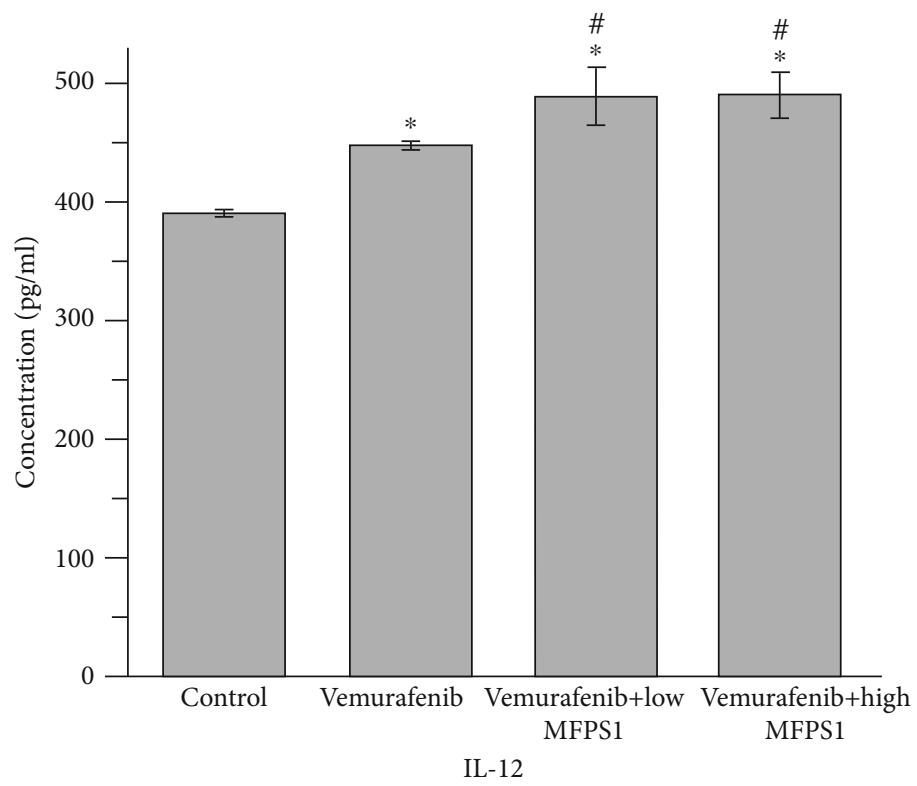

(a)

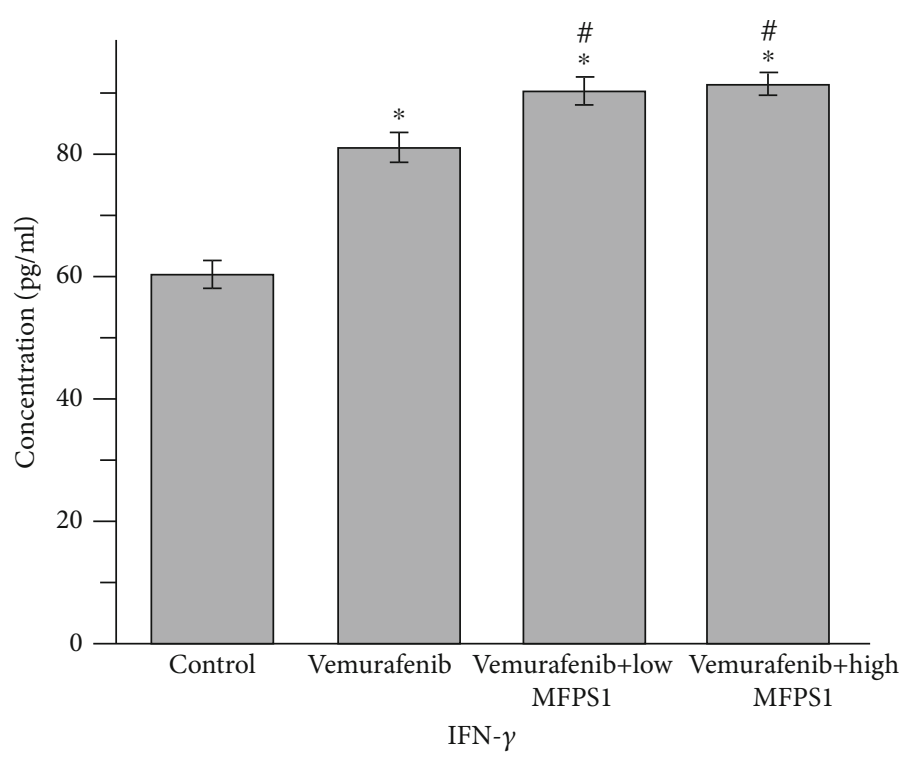

(b)

FIGURE 4: In vivo experiments, MFPS1 enhanced the secretion of cytokines IL-12 and INF- $\gamma$ induced by Vemurafenib in PBMCs of melanoma-bearing mice. (a) The concentration of cytokine IL-12 in PBMCs of melanoma-bearing mice after drug treatment; (b) the concentration of cytokine INF- $\gamma$ in PBMCs of melanoma-bearing mice after drug treatment. * represents the normal group as the control: ${ }^{*} p<0.05$ and ${ }^{* *} p<0.01$; \# represents the Vemurafenib group as the control: ${ }^{*} p<0.05$ and ${ }^{\# \#} p<0.01$.

It has been reported that Th1 ( $\mathrm{T}$ helper cells) lymphocytes and M1 macrophages play a crucial role in antitumor immune surveillance and cytotoxicity [19]. We observed the increased expression of IFN- $\gamma$ and IL-12 in PBMC cells after the combined treatment of MFPS1 and Vemurafenib. On the one hand, IFN- $\gamma$ secreted by Th1 cells plays a role in maintaining the macrophage M1 phenotype, and on the other hand, M1 macrophages release IL-12 to maintain the Th1 phenotype [19]. Therefore, the overexpression of these two cytokines observed in this study suggests that the combination of MFPS1 and Vemurafenib can initiate proinflammatory antitumor immune surveillance via $\mathrm{m} 1 \Phi$.

An increase in CD335+ NK cells was also found in PBMCs treated with MFPS1 and Vemurafenib. It has also been reported that the overexpression of TNF- $\alpha$ can activate NK cells against tumor cells $[20,21]$. In this study, we also showed that the combination of MFPS1 and Vemurafenib can trigger the activation and proliferation of $\mathrm{B}$ cells and $\mathrm{T}$ cells. 

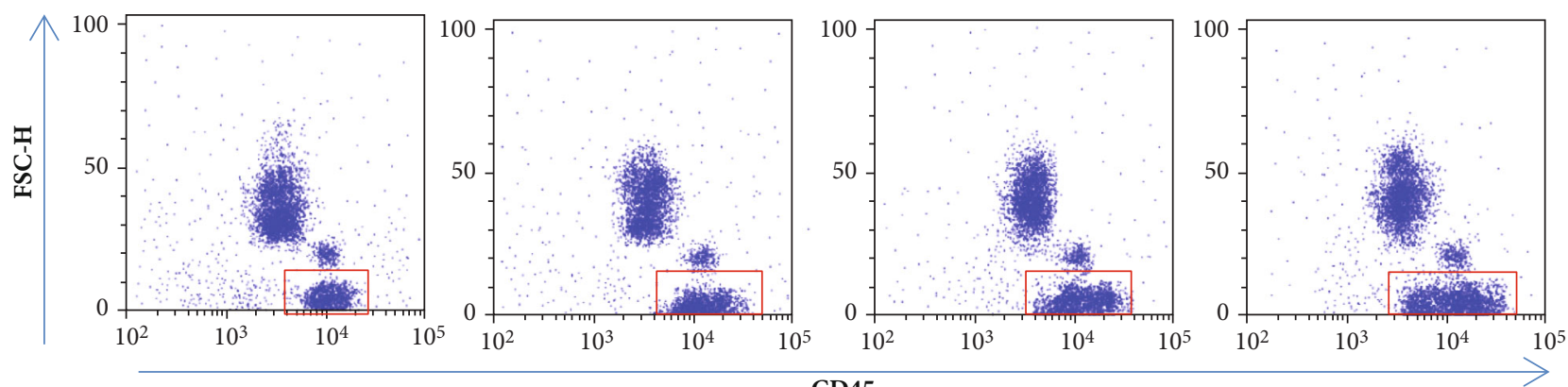

\section{CD45}

(a)

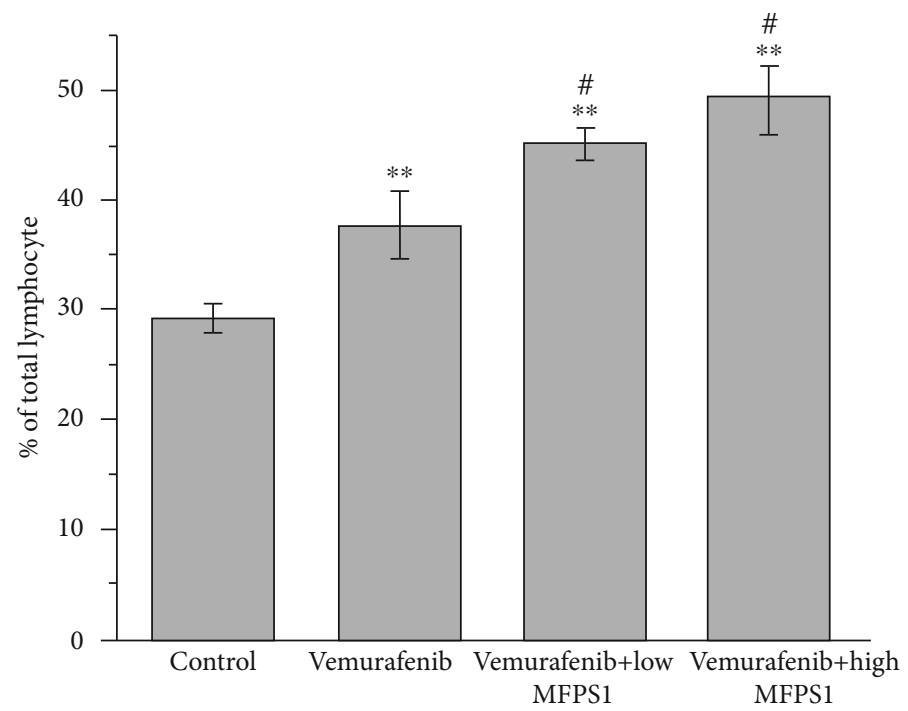

(b)
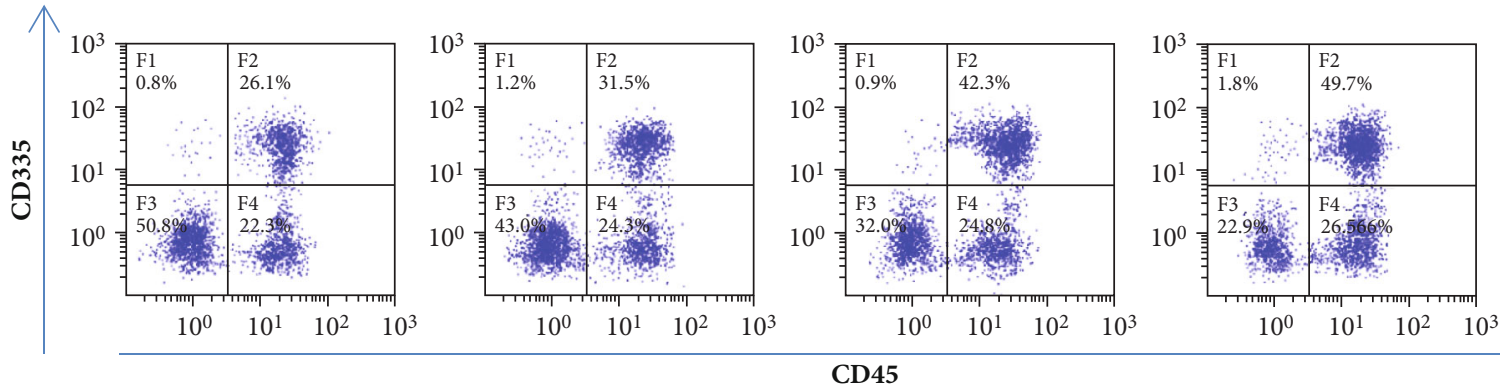

(c)

Figure 5: Continued. 


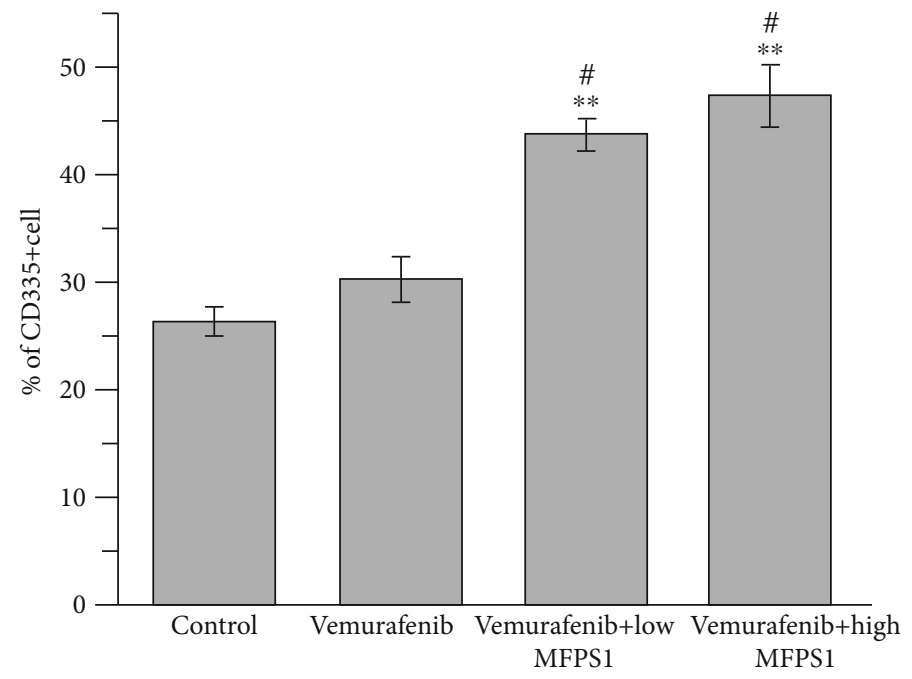

(d)
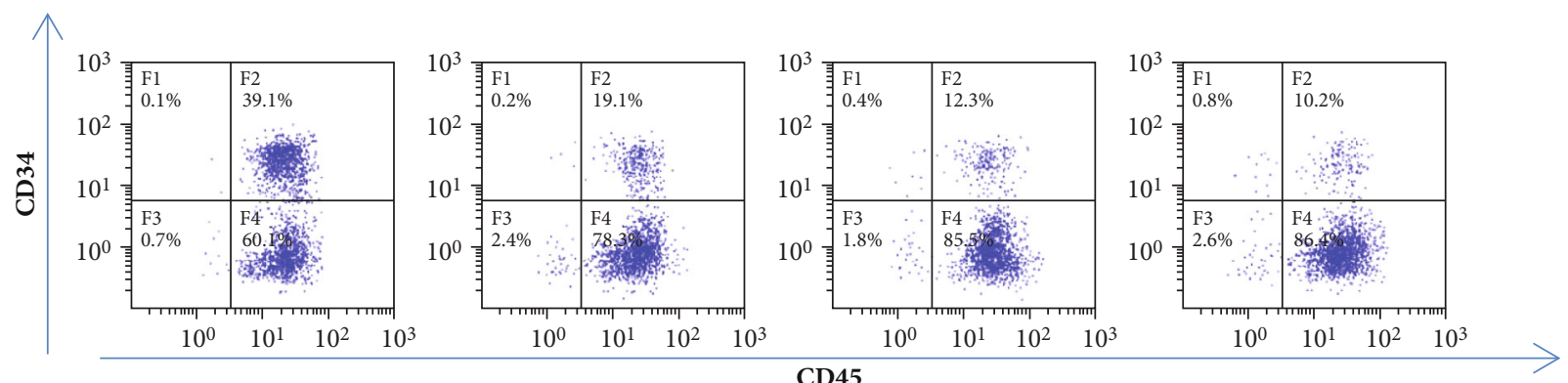

(e)

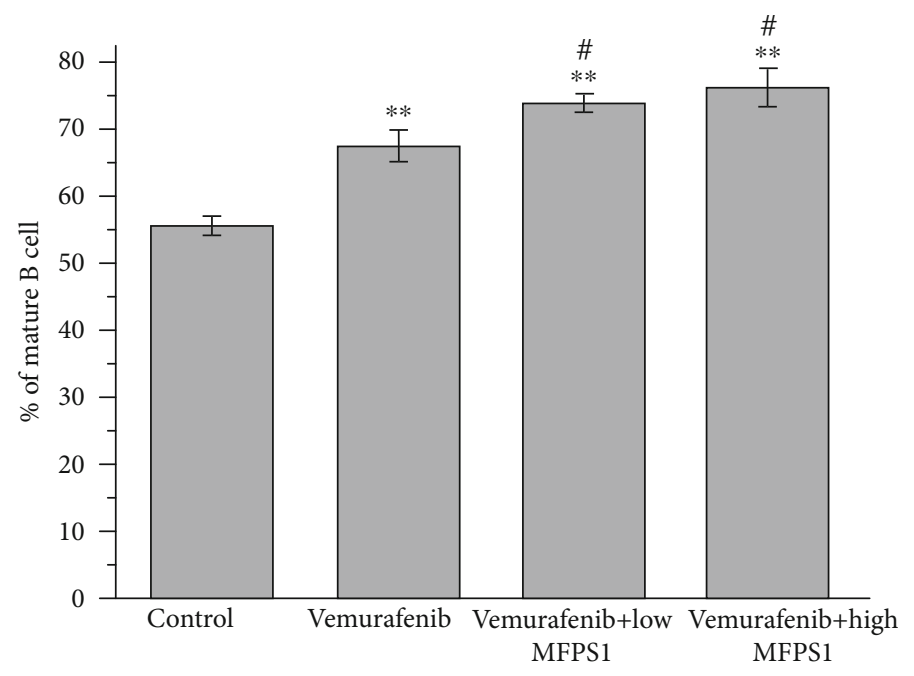

(f)

FIgURE 5: Continued. 


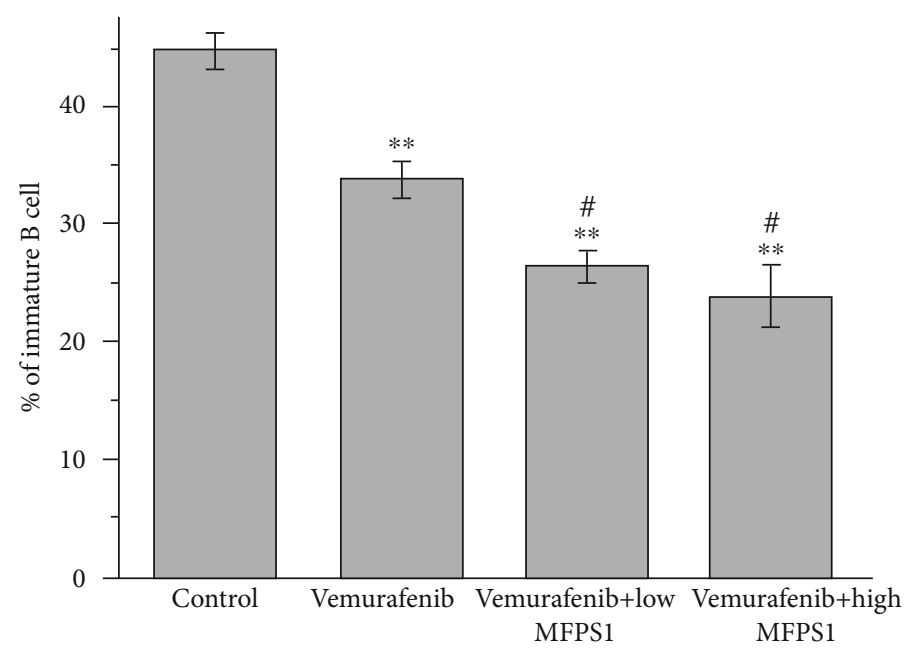

(g)
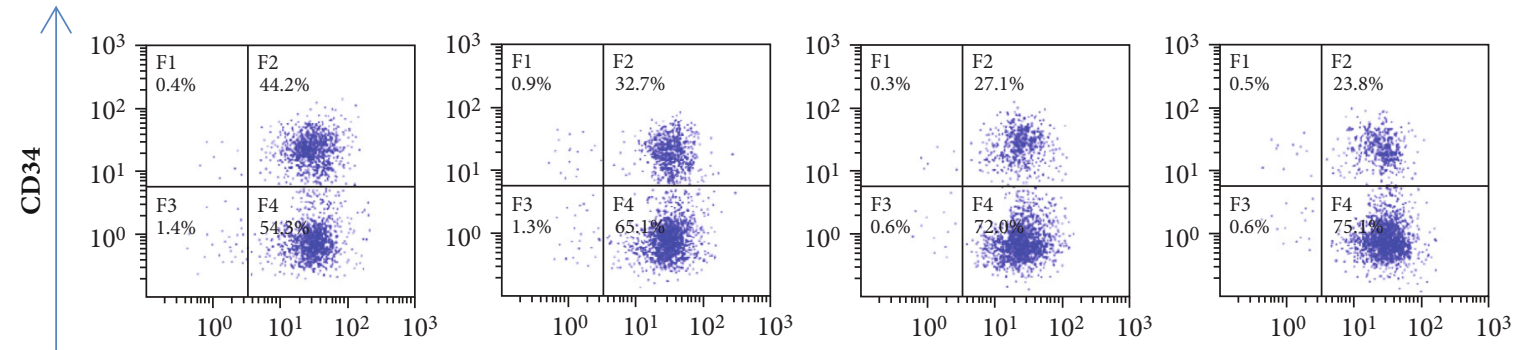

CD19

(h)

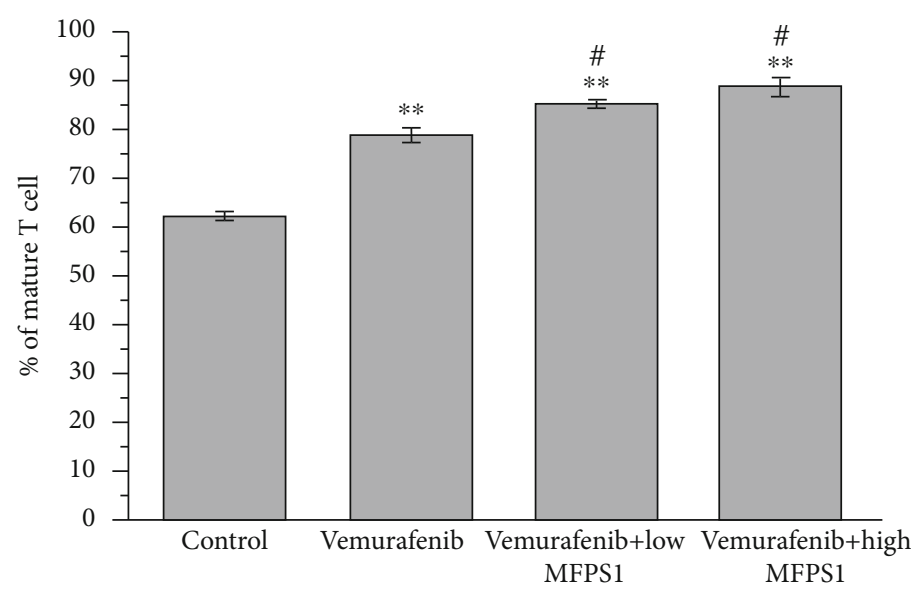

(i)

Figure 5: Continued. 


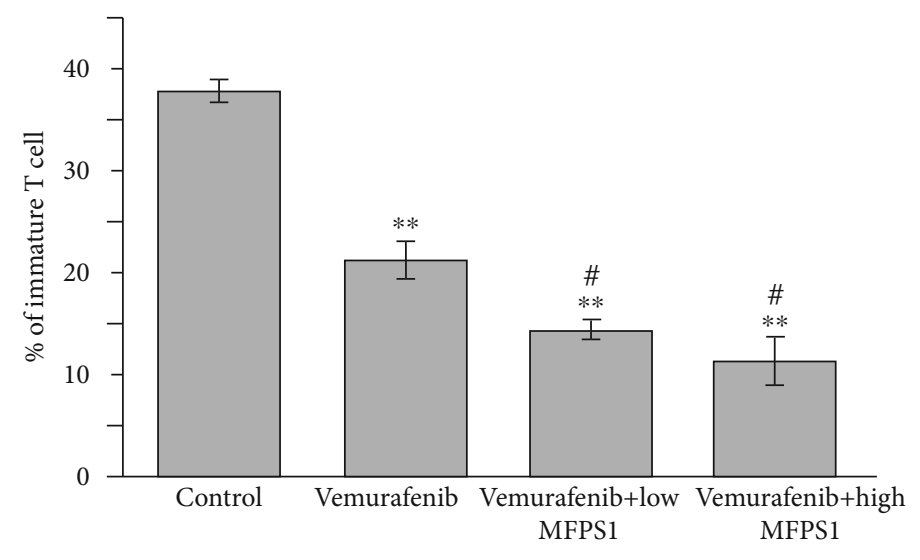

(j)

FIgURE 5: Effect of MFPS1 combined with Vemurafenib on immunomodulatory activity in melanoma-bearing mice. Results of immunophenotypic flow cytometry for a single mouse ( $a, c, e, h) ;(a, b)$ lymphocyte content in PBMCs of melanoma-bearing mice after drug treatment; $(c, d)$ CD335-positive NK cell content in PBMCs of melanoma-bearing mice; (e, f) the content of mature B cells in PBMCs of melanoma-bearing mice (upper right corner in (e)); (e, g) the content of immature B cells in PBMCs of melanoma-bearing mice (lower right corner in (e)); $(\mathrm{h}, \mathrm{i})$ the content of mature $\mathrm{T}$ cells in PBMCs of melanoma-bearing mice (upper right corner in $(h)) ;(h, j)$ the content of immature $\mathrm{T}$ cells in PBMCs of melanoma-bearing mice (lower right corner in (h)). $*$ represents the normal group as the control: ${ }^{*} p<0.05$ and ${ }^{* *} p<0.01$; \# represents the Vemurafenib group as the control: ${ }^{*} p<0.05$ and ${ }^{\# \#} p<0.01$.

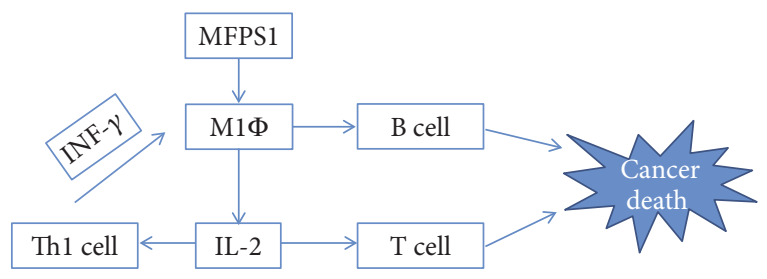

Figure 6: The speculated antitumor mechanism for the combination use of MFPS1 with Vemurafenib.

\section{Conclusion}

Taken together, the combination of MFPS1 and Vemurafenib may initiate proinflammatory antitumor immune surveillance via $\mathrm{m} 1 \Phi$, triggering the activation and proliferation of $\mathrm{B}$ cells and $\mathrm{T}$ cells and enhancing the immunomodulatory activity of melanoma-bearing mice (Figure 6).

\section{Data Availability}

The data used to support the findings of this study are available from the corresponding author upon request.

\section{Conflicts of Interest}

The authors declare that there is no conflict of interest regarding the publication of this paper.

\section{Acknowledgments}

This work supported by the Zhejiang Medical and Health Research Project (2018KY216).

\section{References}

[1] J. Schlom, P. M. Arlen, and J. L. Gulley, "Cancer vaccines: moving beyond current paradigms," Clinical Cancer Research, vol. 13, no. 13, pp. 3776-3782, 2007.

[2] C. J. Wheeler, A. Das, G. Liu, J. S. Yu, and K. L. Black, "Clinical responsiveness of glioblastoma multiforme to chemotherapy after vaccination," Clinical Cancer Research, vol. 10, no. 16, pp. 5316-5326, 2004.

[3] L. Apetoh, S. Ladoire, G. Coukos, and F. Ghiringhelli, "Combining immunotherapy and anticancer agents: the right path to achieve cancer cure?," Annals of Oncology, vol. 26, no. 9, pp. 1813-1823, 2015.

[4] M. Lemieszek and W. Rzeski, "Anticancer properties of polysaccharides isolated from fungi of the Basidiomycetes class," Contemporary Oncology, vol. 16, no. 4, pp. 285-289, 2012.

[5] C. Sanchez, "Reactive oxygen species and antioxidant properties from mushrooms," Synthetic and Systems Biotechnology, vol. 2, no. 1, pp. 13-22, 2017.

[6] X. Huang and S. Nie, "The structure of mushroom polysaccharides and their beneficial role in health," Food \& Function, vol. 6 , no. 10, pp. 3205-3217, 2015.

[7] X. Meng, H. Liang, and L. Luo, “Antitumor polysaccharides from mushrooms: a review on the structural characteristics, antitumor mechanisms, and immunomodulating activities," Carbohydrate Research, vol. 424, pp. 30-41, 2016.

[8] S. I. Grivennikov, F. R. Greten, and M. Karin, "Immunity, inflammation, and cancer," Cell, vol. 140, no. 6, pp. 883-899, 2010.

[9] S. Wasser, "Medicinal mushrooms as a source of antitumor and immunomodulating polysaccharides," Applied Microbiology and Biotechnology, vol. 60, no. 3, pp. 258-274, 2002.

[10] D. Li, W. Xu, P. Li et al., "Self-targeted polysaccharide prodrug suppresses orthotopic hepatoma," Molecular Pharmaceutics, vol. 13, no. 12, pp. 4231-4235, 2016. 
[11] M. F. Moradali, H. Mostafavi, S. Ghods, and G. A. Hedjaroude, "Immunomodulating and anticancer agents in the realm of macromycetes fungi (macrofungi)," International Immunopharmacology, vol. 7, no. 6, pp. 701-724, 2007.

[12] C. Garbe and T. K. Eigentler, "Vemurafenib," Recent Results in Cancer Research, vol. 211, pp. 77-89, 2018.

[13] L. D. Gutierrez-Castaneda, J. A. Nova, and J. D. Tovar-Parra, "Frequency of mutations in BRAF, NRAS, and KIT in different populations and histological subtypes of melanoma: a systemic review," Melanoma Research, p. 1, 2019.

[14] G. Kim, A. E. McKee, Y. M. Ning et al., "FDA approval summary: vemurafenib for treatment of unresectable or metastatic melanoma with the BRAFV600E mutation," Clinical Cancer Research, vol. 20, no. 19, pp. 4994-5000, 2014.

[15] G. Bollag, J. Tsai, J. Zhang et al., "Vemurafenib: the first drug approved for BRAF-mutant cancer," Nature Reviews. Drug Discovery, vol. 11, no. 11, pp. 873-886, 2012.

[16] K. K. Maity, S. Patra, B. Dey et al., "A heteropolysaccharide from aqueous extract of an edible mushroom, Pleurotus ostreatus cultivar: structural and biological studies," Carbohydrate Research, vol. 346, no. 2, pp. 366-372, 2011.

[17] P. Slobbe, A. D. Windhorst, K. Adamzek et al., "Development of $\left[{ }^{11} \mathrm{C}\right]$ vemurafenib employing a carbon-11 carbonylative Stille coupling and preliminary evaluation in mice bearing melanoma tumor xenografts," Oncotarget, vol. 8, no. 24, pp. 38337-38350, 2017.

[18] A. K. MacLeod, L. A. McLaughlin, C. J. Henderson, and C. R. Wolf, "Activation status of the pregnane $\mathrm{X}$ receptor influences vemurafenib availability in humanized mouse models," Cancer Research, vol. 75, no. 21, pp. 4573-4581, 2015.

[19] O. A. W. Haabeth, B. Bogen, and A. Corthay, "A model for cancer-suppressive inflammation,” OncoImmunology, vol. 1, no. 7, pp. 1146-1155, 2014

[20] B. Burkholder, R. Y. Huang, R. Burgess et al., "Tumor-induced perturbations of cytokines and immune cell networks," Biochimica et Biophysica Acta (BBA) - Reviews on Cancer, vol. 1845, no. 2, pp. 182-201, 2014.

[21] E. Vivier, S. Ugolini, D. Blaise, C. Chabannon, and L. Brossay, "Targeting natural killer cells and natural killer T cells in cancer," Nature Reviews. Immunology, vol. 12, no. 4, pp. 239-252, 2012. 


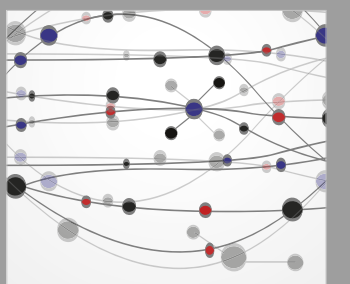

The Scientific World Journal
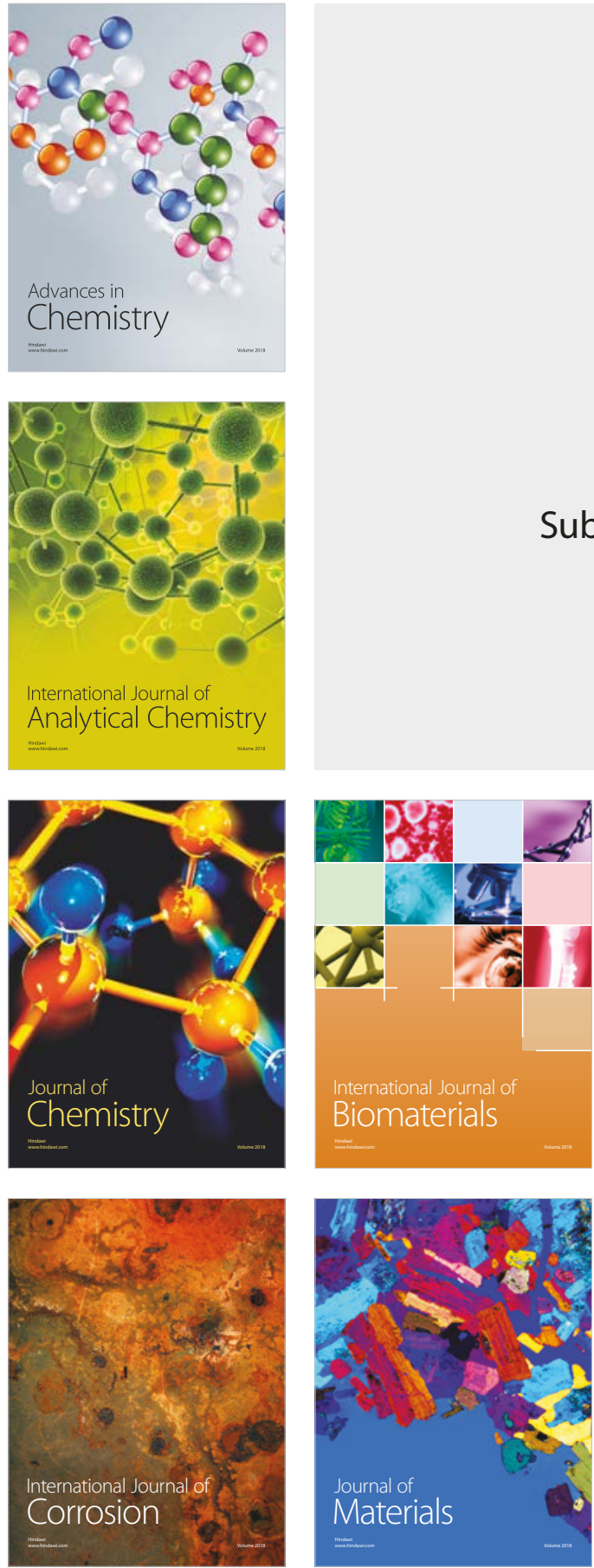

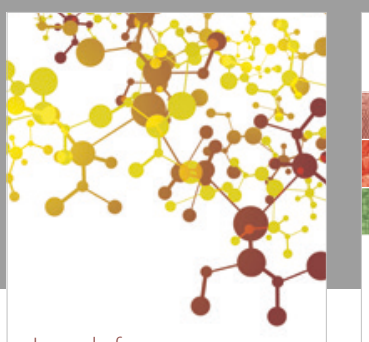

Journal of

Applied Chemistry
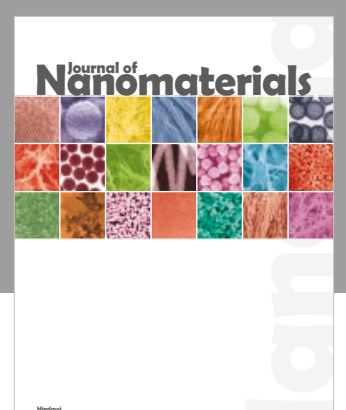

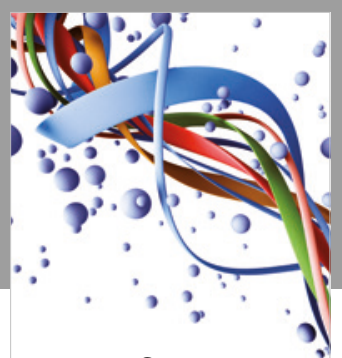

Scientifica

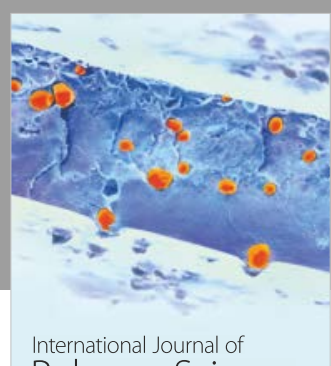

Polymer Science

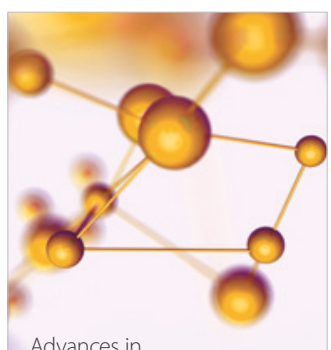

Physical Chemistry
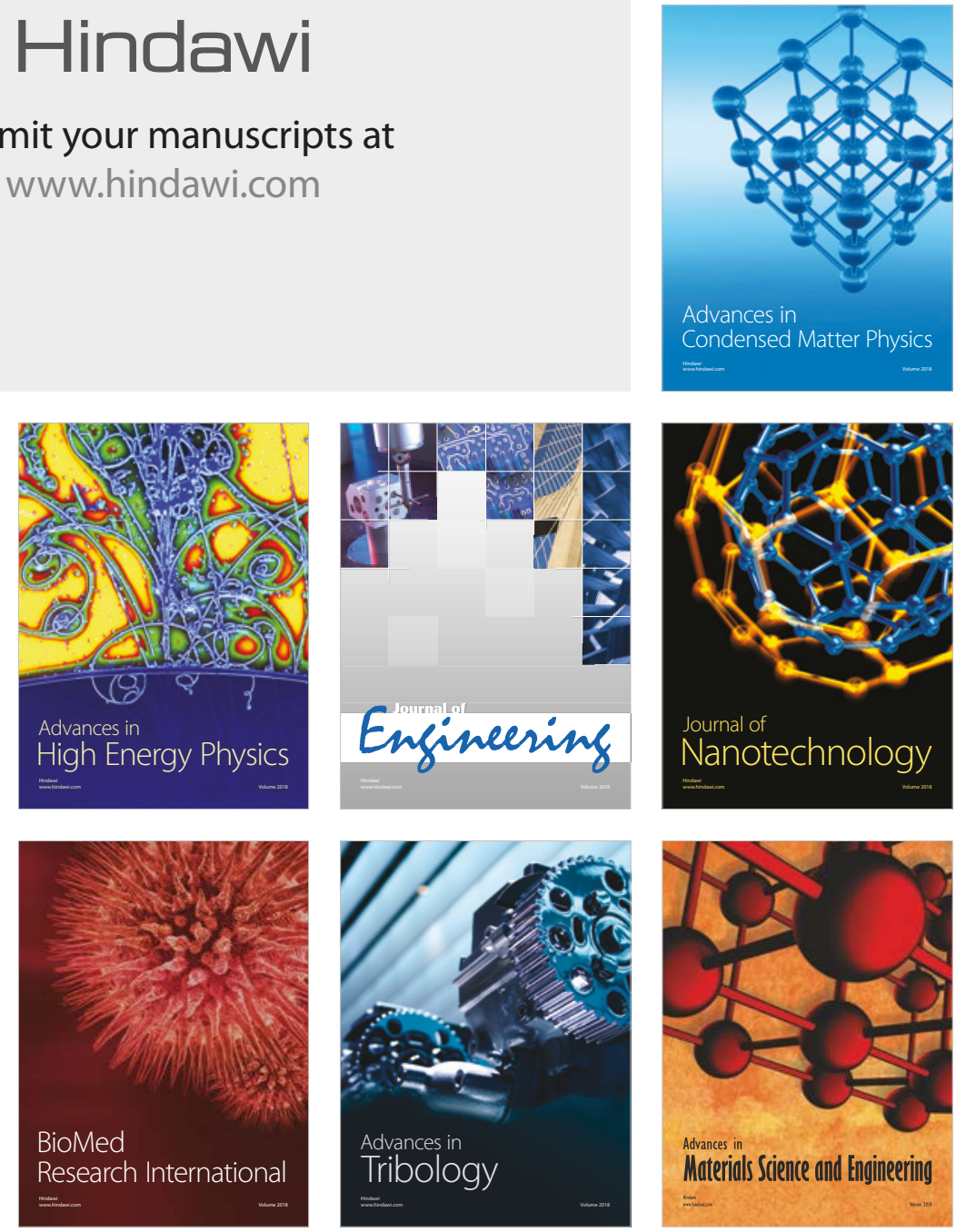